\title{
Aducanumab: Appropriate Use Recommendations
}

\author{
P. Scheltens ${ }^{1}$, E.G.B. Vijverberg ${ }^{1,2}$

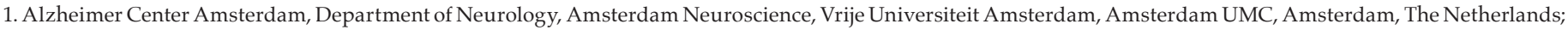 \\ 2. Brain Research Center, Amsterdam, The Netherlands

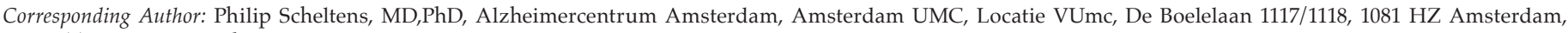 \\ T: +31 (0)20 4440816, p.scheltens@amsterdamumc.nl
}

J une 7, 2021 will not likely be forgotten soon by many Alzheimer Disease (AD) researchers. To paraphrase a famous quote: "a small step for man, but a giant leap forwards for the field". That day, Aduhelm ${ }^{\mathrm{TM}}$ (aducanumab) was approved by the US Food and Drug administration (FDA), because of its profound effect on amyloid plaques as shown by amyloid PET as a surrogate marker. Although this decision was not met with great enthusiasm uniformly, the general feeling during a 4 hour webinar hosted by the Alzheimer Association (1) was that the benefits seem to outweigh the risks, but proper guidance was needed on who would be eligible for treatment, because all attendees felt the label was far too broad and unspecific. The latter was amended on july 8, jointly by FDA and Biogen to more accurately reflect the eligible patient population that was studied in the Phase III program.

In this issue of JPAD, Cummings and colleagues report Appropriate Use guidelines constructed by an Expert Panel (2). Not surprisingly, The Expert Panel recommends that the appropriate use of aducanumab in real-world clinical practice should pragmatically mimic the use of aducanumab in the EMERGE and ENGAGE clinical trials that led the FDA to approve aducanumab. Importantly, not mentioned explicitly in the label, they stress that a patient who is considered for treatment with Aduhelm, should be amyloid positive, using any of the approved amyloid ligands, visually read by an experienced nuclear medicine physician, or by having abnormal CSF levels. We would like to specify this by stating that both CSF abeta 1-42 and p-tau 181 should be abnormal (or an abnormal ratio ptau/abeta), in agreement with the biological definition of $\mathrm{AD}$ (3). In order to lower the number of patients undergoing expensive PET scanning or invasive lumbar punction procedures, the availability of plasma tests to prescreen patients will be highly valuable (4). This is becoming a reality quite soon and will reduce costs for the healthcare system as well.

The Expert Panel stresses the patiënt-centered discussion around the decision to start treatment and assessing APOE status. The latter is important given the higher risk of ARIA in E4 carriers but even more because of a possible even higher treatment effect (5). This shared decision making around these important issues requires more time and effort from an experienced AD physician, most often a neurologist, than previously needed and thought (6).

The Expert panel makes important recommendations to monitor patients for ARIA using a similar scheme as was employed in the trials. It may be emphasized that ARIA is an MRI phenomenon that remained asymptomatic in $75 \%$ of the participants in the trials. It is conceivable that this is actually lower in real world practice, since in the trials patients were specifically asked for side effects. That said, the treating physician should be alert for side effects and should taper the treatment upon the appearance of ARIA, as per the criteria laid out by Cummings et al. In case of severe symptomatology, treatment with steroids is highly effective, but rarely needed.

One category of patients that were not included in the trials and not mentioned in the citeria, but who may appeal for treatment to their phsyicians is familial AD: patients carrying a PSEN1, 2 or APP mutation. When symptomatic and amyloid positive, they may be eligible for treatment and the same recommendations will apply to them.

The Expert Panel has given timely advice and it is hoped that all sites around the US will apply these recommendations in order to minimize regional differences, to optimize treatment and monitoring with an expensive drug in a vulnerable population of patients and it forms a solid basis for future treatment with other monoclonal antibodies for AD. When EMA approves aducanumab in the EU, these Appropriate Use guidelines will help formulating similar advice to EU clinicians, to secure the right patient is treated in the most optimal way.

\section{References}

1. https://www.alz.org/research/for_researchers/grants/medsci-webinars/ on-demand-research-webinars

2. Cummings J., Aisen P., Apostolova L.G. et al. Aducanumab: Appropriate Use Recommendations. J Prev Alz Dis 2021;4(8):398-410; DOI: http:/ /dx.doi. org/10.14283/jpad.2021.41

3. Jack CR Jr, Bennett DA, Blennow K, Carrillo MC, Dunn B, Haeberlein SB, Holtzman DM, Jagust W, Jessen F, Karlawish J, Liu E, Molinuevo JL, Montine T, Phelps C, Rankin KP, Rowe CC, Scheltens P, Siemers E, Snyder HM, Sperling R; Contributors. NIA-AA Research Framework: Toward a biological definition of Alzheimer's disease. Alzheimers Dement. 2018 Apr;14(4):535-562.

4. Thijssen EH, Verberk IMW, Vanbrabant J, Koelewijn A, Heijst H, Scheltens P, van der Flier W, Vanderstichele H, Stoops E, Teunissen CE. Highly specific and ultrasensitive plasma test detects Abeta(1-42) and Abeta(1-40) in Alzheimer's 
disease. Sci Rep. 2021 May 6;11(1):9736.

5. Briefing document Biogen and FDA, November 6, 2020

6. Fruijtier AD, Visser LNC, Bouwman FH, Lutz R, Schoonenboom N, Kalisvaart K, Hempenius L, Roks G, Boelaarts L, Claus JJ, Kleijer M, de Beer M, van der
Flier WM, Smets EMA. What patients want to know, and what we actually tell them: The ABIDE project. Alzheimers Dement (N Y). 2020 Dec 16;6(1):e12113.

How to cite this article: P. Scheltens, E.G.B. Vijverberg. Aducanumab: Appropriate Use Recommendations. J Prev Alz Dis 2021;4(8):412-413; http:/ / dx.doi.org/10.14283/jpad.2021.45 